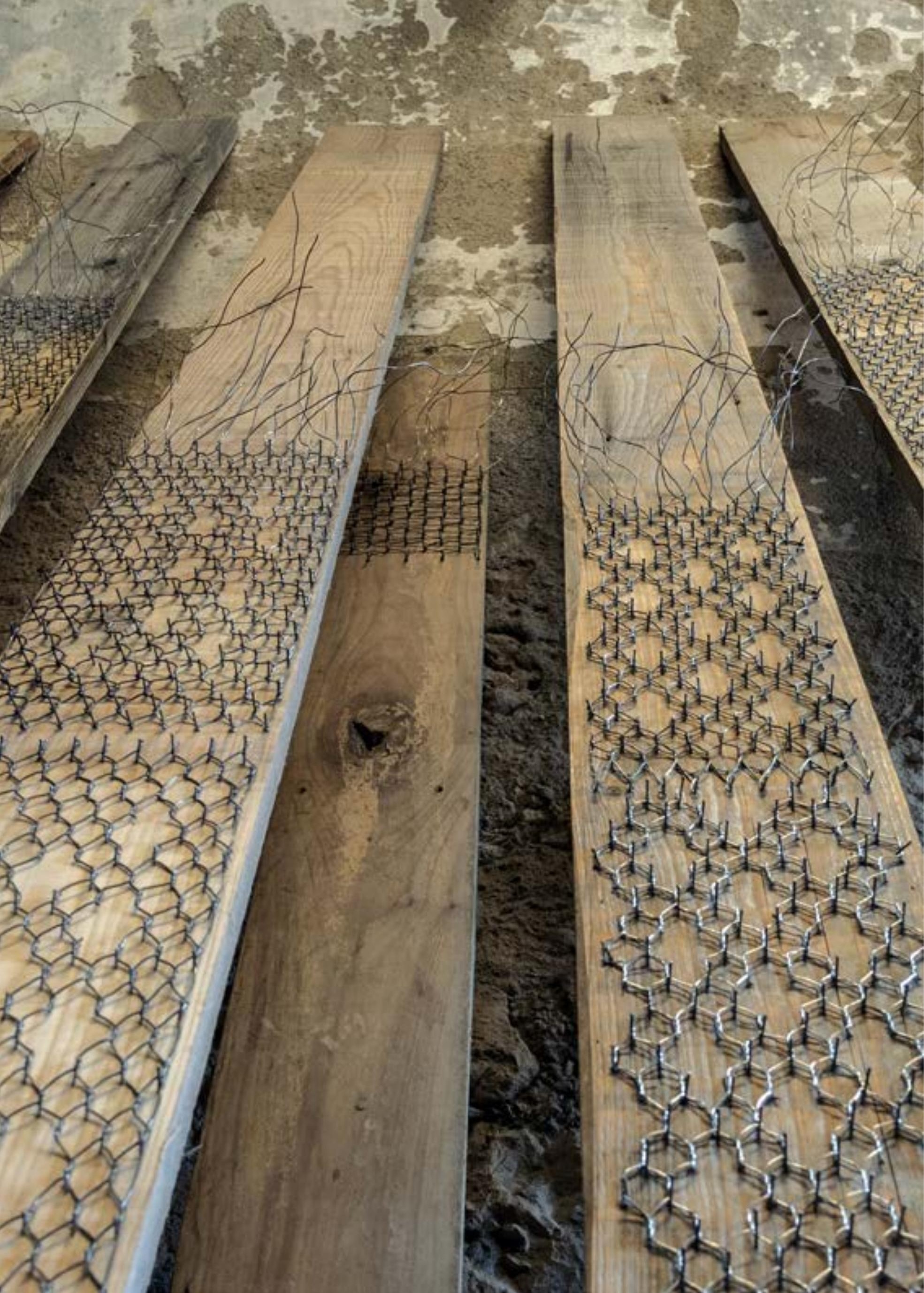




\title{
Efficacia dell'arte, strategie di rete e approccio trasformativo a paesaggio e patrimonio alpino
}

Effectiveness of art, network strategies and transformative approach to landscape and alpine heritage

\begin{abstract}
Ever since 2011, Dolomiti Contemporanee (DC) has been operating on the contemporary identity of the mountain, and on the state of the Landscape, as well as the cultural, historic, and architectural Heritage inside the region of UNESCO's Dolomites.

Its research takes shape in the reactivation of large, issue-heavy industrial archaeology sites and compounds: former factories, former social villages, iconic architectural creations, with a great historical or aesthetic value, abandoned and underutilized, immersed in the powerful nature of the Dolomitic region.

DC works on the redefinition of the mountain's identity, building re-innovative and thematic critical images. In this sense, we refuse to recognize as an acceptable identity for the Alpine landscape the hotchpotch of stereotypical reductions which deliver a bland and reified vision of it, one that almost always involves plain and simple economic and touristic exploitation of the asset, to the detriment of its real potential's nourishment.

DC's practice puts at the centre the need for re-enhancement and functional re-use of a few exceptional sites, which must be re-processed and re-activated.

It is a responsible necessity of care and an opportunity for the regeneration of extraordinary underdeveloped sources of potential at the same time.

Contemporary art, innovation culture, network strategies, those are some of the "techniques" through which such sites, so important in the past and now lifeless, are tackled, and morphed into cultural and artistic production centres, finally operative again, engines able to represent and provide new value to the territory.
\end{abstract}

\section{Gianluca d'Incà Levis}

Born in 1969. Graduated in architecture at the IUAV of Venice.

Creator and curator of Dolomiti

Contemporanee (2011), and Progettoborca, director of Spazio di Casso at Vajont. At the center of research, a core of renewal practices for landscape and mountain, which include the regeneration of large problematic sites.
Keywords

Dolomites, legacy, reuse, regeneration, contemporary art. 
Dolomiti Contemporanee (www.dolomiticontemporanee.net - d'ora innanzi $\mathrm{DC}$ ) è un progetto ancor meglio processo - che, attraverso una visione spiccatamente operativa, e aggressiva quanto basta, ripensa fattivamente al valore e all'identità delle cose del paesaggio e della montagna, quelle cose che non di rado vengono trattate come un tavolo da pranzo, salame appeso, bene di consumo (seppur periferico, e in ciò persino marginale), invece che come un valore essenziale (e in ciò sempre centrale).

DC non sorge su alcuna teoria pregressa, né costituisce un modello agilmente replicabile: per questo preferiamo processo a progetto. Si tratta di una visione, di un approccio, di una tecnica: di cura e di scasso, trasvalutativi, del territorio, del paesaggio, e delle sue risorse trascurate.

Riteniamo che - se fossimo persone serie, e non soggetti approssimativi (trafficanti o trafficoni) il potenziale d'ogni cosa, e dunque anche della montagna (noi viviamo qui: le cose si fanno dall'interno) andrebbe costantemente fatto emergere, correttamente sviluppato e, laddove necessario, riprocessato (invece che ancorato a intransitive nostalgie passivanti).

E riteniamo che ciò possa e debba essere fatto attraverso l'applicazione di forze positive (e perciò oppositive, quando serve), e non, ad esempio, attraverso l'uso delle deboli o debolissime categorie merceologiche, nate-fossili, della Montagna-Mangiatoia-Mercato (è piena la montagna di triple $\mathrm{M}$ inflattive), spesso praticate dalle confuse rapide ancelle del Mero spirito del Marketing al Microonde.

Ma, d'altro canto, ci sono anche gli incoronamenti (pseudo)letterari da trivio, anch'essi capaci di incatenare la montagna al consumo istantaneo di un pasto a debolissimo contenuto proteico. Le diete privative nuocciono, vanno contrastate. Era meglio Prometeo: da quel fegato dilaniato cola potassio sulle rocce.

La montagna è alta, dura, frappone ostacoli, muove l'ingegno alle soluzioni. Pareti, bivacchi, paesi inerpicati, strade impuntate, sentieri e boschi (schiantati, con Tempesta Vaia). Uno spazio così configurato è il luogo ideale, per la ricerca e la sperimentazione dei moduli tutti: sociali, abitativi, costruttivi, culturali, di sensibilità, di paesaggio e di passaggio, d'infrastruttura, e così via, direbbe Caulfield.

Dove si può salire, retta talvolta è la via della ricerca. La massima pendenza scoraggia i pianeggiatori mentali - mica sempre però, mica sempre.

E però, per agir bene, si diceva, c'è anche da contrastare, da scassare: quei meccanismi fatui, sperequativi, reazionari, irresponsabili, appropriativi, legati alle logiche di fruizione-consumo della qualità del bene. Come anche le narrazioni da cassetta.

Diciamolo già: alcuni beni selvareghi sono tali perché intonsi, o perlomeno intatti. Se ci porti l'uomo in massa, ad esempio, una cima, come un atollo, non son più quel bene che erano, ma un salame sul tagliere.

E dunque? Intendiamo con ciò ablare le Dolomiti, renderle indisponibili, levarle dai flussi, conservarle, immobilizzarle? In tal senso qualche anno fa l'amico nostro Marc Augé contestava la logica imbalsamatrice dei riconoscimenti Unesco.

Conservare non è sufficiente. D'altro canto, consumare e spremere è indegno e miope.

Noi vogliamo una coscienza operativa del sistema, non a sola tutela.

Insistiamo ancora un attimo sulle cose inappropriate, sulle male coltivazioni.

Se, ad esempio, sulla montagna si scrivono libracci o libruzzi, i coacervi commerciali di banalità e cliché, ciò genera cattiva comunicazione e cattiva identità: questo è grave, un tradimento del valore, a questo ci opponiamo.

Se un Sindaco, quando ragiona su sviluppo e crescita del proprio territorio montano, è capace di pensare solo a vernacolo e tradizione, sagra e polenta e alpini (attenzione: con tutto ciò noi sappiamo lavorare bene, attraverso le pratiche del contemporaneo), alle immagini retroverse e "nostralgiche" degli avi, alle vacche all'alpeggio e al fischio della marmotta, cioè a tutto l'arsenale retorico di immagini istantanee con cui si usa nutrire branchi di turisti inconsapevoli: con questo Sindaco non lavoriamo. 


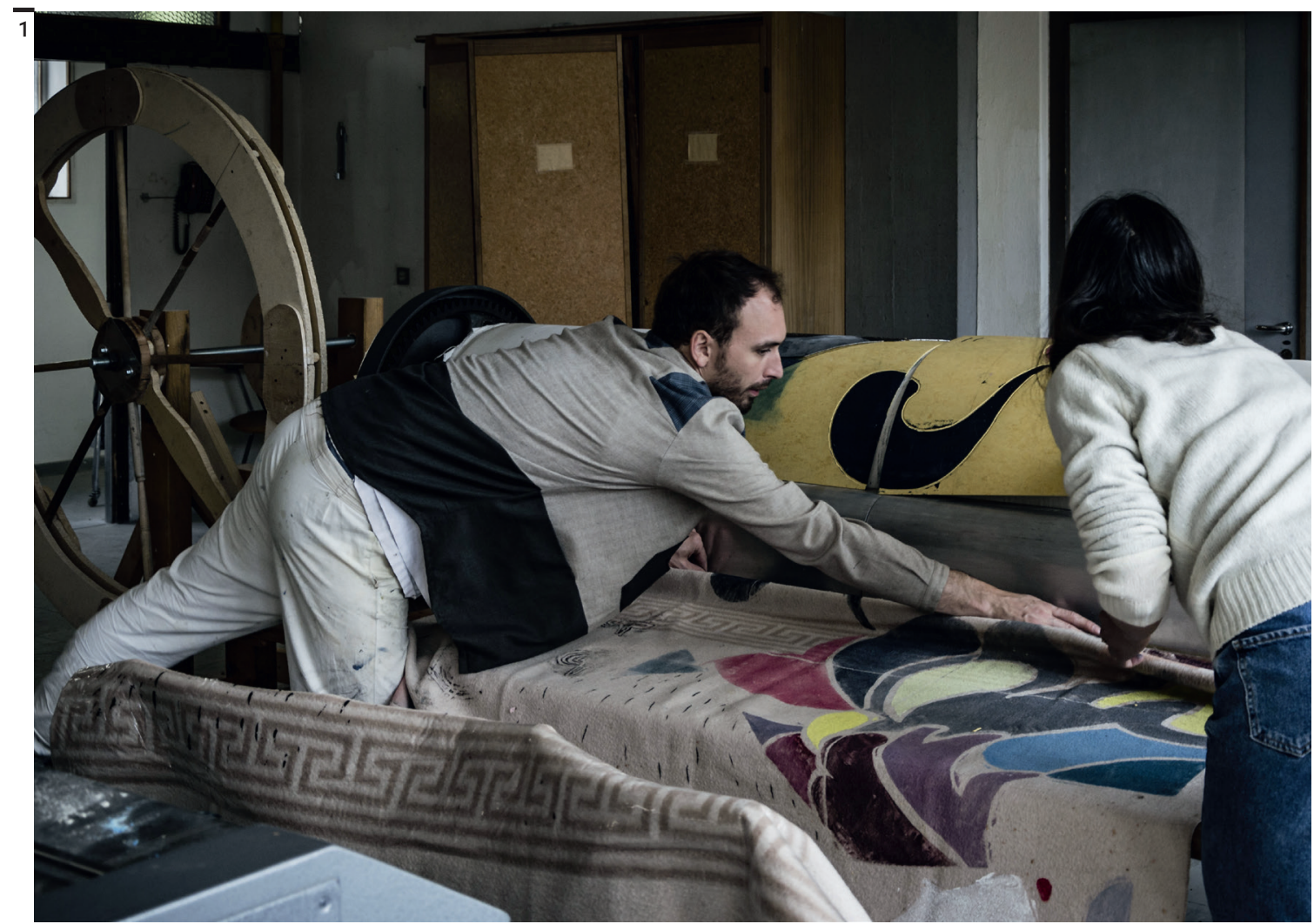

$\overline{2}$

Figg. 1-2

Progettoborca. Gli artisti Sofia Bonato

e Matteo Valerio lavorano alla stampa

sperimentale

rigenerando vecchie

macchine inutilizzate

della Colonia di

Borca. Qui, una

vecchia stiratrice

industriale viene

usata per stampare

capi unici (foto

Nicola Noro).

$\overline{2}$

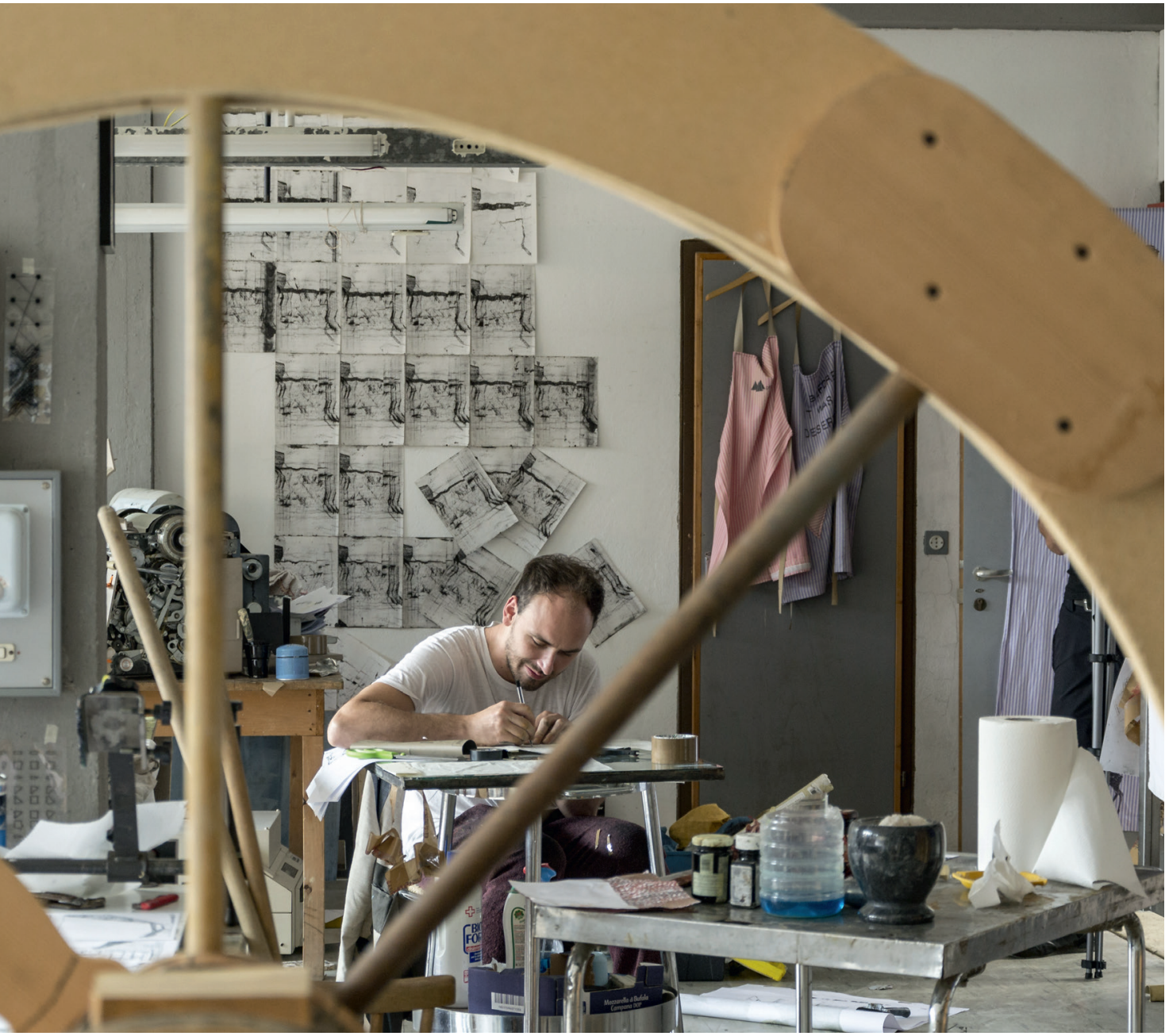


Non c'è un turista, questo bisogna dire ai contabili del mercato-montagna: prima di lui c'è un uomo.

Nutri l'uomo, prima del turista: dagli del buon cibo.

Non ridurre l'uomo a fruitore-compratore, non operare su di un pubblico indifferenziato.

Altrimenti, sei un pizzicagnolo, e non sai far la cultura della tua terra: che è anche la mia (e ciò è pericoloso, crea nocumento).

L'attitudine ad una buona attenzione va costruita: la cultura è formazione.

Che fare dunque? O meglio: cosa facciamo noi?

$\mathrm{Al}$ posto della montagna imbandita (piatto montagna) preferiamo la montagna-cantiere-generativo, spazio dell'esplorazione e dei pionieri: ecco l'alpinismo culturale.

DG nasce nel 2011.

Il progetto traguarda il patrimonio cosiddetto, ambientale, d'architettura, storico e culturale, della regione dolomitica. In particolare, nei primi anni, si è operato nella montagna veneta e friulana (Belluno, Agordino, Cadore, Vajont).

Si esplora il territorio, e si compie un'accurata ricognizione dei siti dismessi o sottoutilizzati, come ex opifici, ex villaggi, strutture o architetture emblematiche o di particolare rilevanza storica, o di intere porzioni di territorio: tutti gravati da criticità e necessità, di progetto e d'uso.

L'obiettivo è quello di trasformare questi siti fer$\mathrm{mi}$, che costituiscono intatte e improcessate risorse territoriali, in cantieri dell'elaborazione e della produzione culturale, all'interno dei quali, attraverso l'idea e la pratica, si riesca da un lato a coltivare un'idea della montagna non stucchevole e retroversa, e invece proiettata (in avanti); dall'altro, a immaginare per questi luoghi inerti nuove identità e funzioni d'uso, rigenerandoli.

Come si capisce, i due obiettivi, posti a scala diverse, coincidono: si tratta di ridefinire costante-

Figg. 3-5

PbLab è un progetto di rebranding. Gli artisti Anna Poletti e Giorgio Tollot hanno trasformato le vecchie coperte di Eni, marcate con il cane a sei zampe e allora realizzate da Lanerossi (oggi Gruppo Marzotto), in capi vintage decorati a mano, con motivi e temi mutuati dalle architetture di Edoardo Gellner (foto Nicola Noro (3) e Giacomo De Donà). mente l'essenza di una serie di beni-risorsa che però, esauritasi la loro prima identità, allo stato attuale giacciono, immobili, senza più concorrere allo sviluppo del territorio.

In otto anni di attività, DC ha affrontato una ventina di siti, secondo modalità e strategie diverse, nell'obiettivo e nel periodo.

Lo strumento principe di questa politica rinnovative e rigenerativa è il contemporaneo.

Il contemporaneo non è un trastullo: l'arte contemporanea è uno strumento della formazione, intellettuale, culturale, estetica.

Fabbriche abbandonate da decenni, spesso addirittura restaurate, e però mai riavviate, con grave sperpero. Come ad esempio l'ex polo chimico Montedison di Sass Muss, o l'ex fabbrica di occhiali Visibilia, entrambe nel bellunese.
Siti sempre eccezionali, nella storia, nell'estetica, nel rapporto col paesaggio montano, come il Castello di Andraz a Livinallongo del Col di Lana.

Questi siti, cimiterizzati, spenti, o nella necessità di un riconcepimento d'identità e d'uso, sono tutti spettacolari, immersi nel formidabile ambiente dolomitico.

Chiunque li veda, ne intende il valore cosiddetto.

Ma cosa significa valore, se $\mathrm{i}$ siti in realtà sono in stallo, immoti, come ferri-da-stiro del paesaggio? Valore è dunque un'aura, un'ombra del passato? Un attributo teorico? Come mai, nella pratica, l'uomo, utilizzando gli strumenti convenziona-
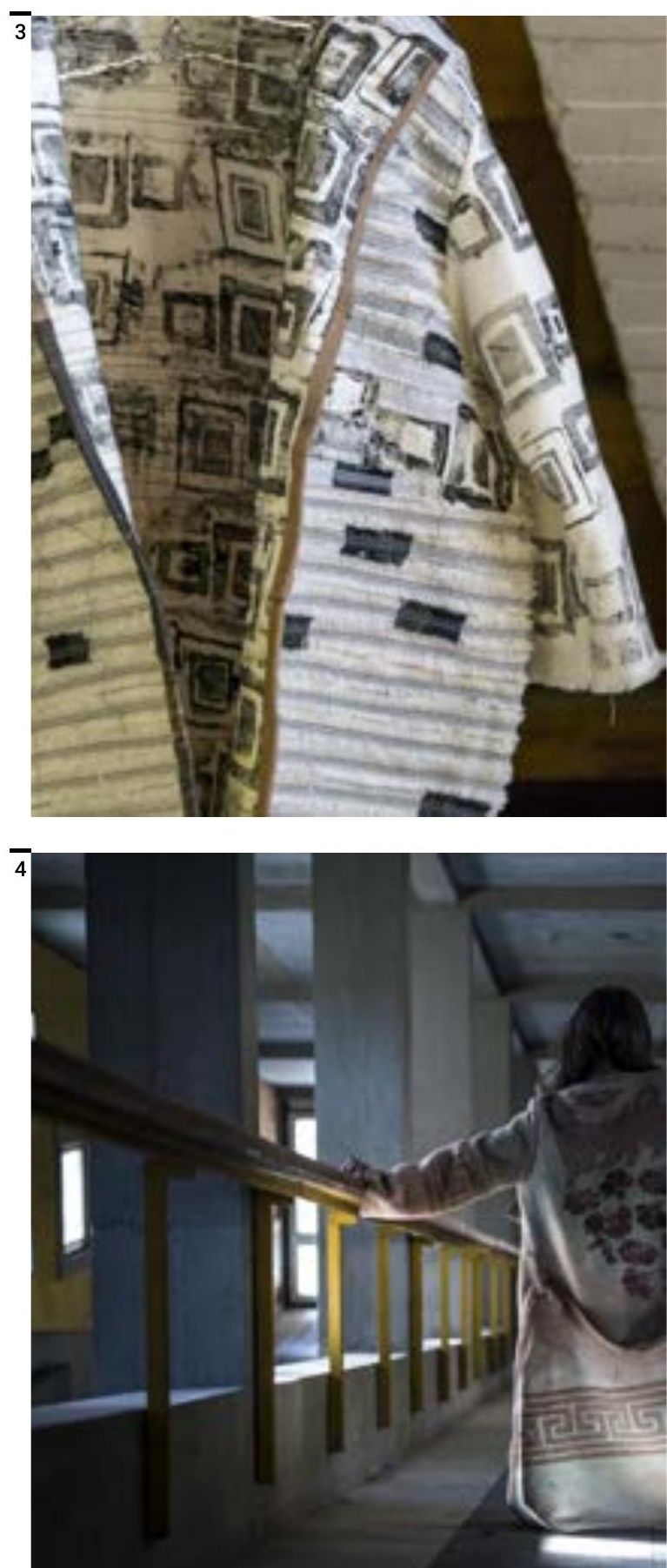
li della politica, della governance, dell'impresa (o della speculazione), le categorie e i gerghi dell'architettura e dell'archeologia industriale, non l'ha saputo ri-agire correttamente, questo preteso valore?

È l'abissale distanza tra valore residuale e valore reale e d'uso, quella che si vuol colmare, per far corrispondere il potenziale alla funzione.

I siti che inquadriamo non presentano deficit propri: sono intatti, potenti, disponibili.

Cosa manca? Un pensiero diritto, e una pratica diretta. Il deficit è dell'uomo: è cognitivo, immaginativo.

E se non pensi bene, come potrai operare mai?
I siti vanno ripensati bene e resi fruibili attraverso un'idea sensata, non agghindati e reimessi sul mercato, sperando che qualcuno se li compri (infatti, nessuno li ha comprati).

Ed è chiaro come DC non si occupi di neoimmobiliarismo cultuale, né di captazione commerciale. Così operiamo noi: attorno ad ogni sito latente, costruiamo una rete di aderenze, spingendo sull'idea-necessità, e sulla possibilità concreta, della sua rigenerazione. All'impresa, associamo gli enti d'area, pubblici e privati, le imprese, interi strati della socialità. Cerchiamo, subito, la condivisione dell'intento, dapprima a livello locale, poi, per cerchi sempre più ampi, centrifughi, all'esterno.
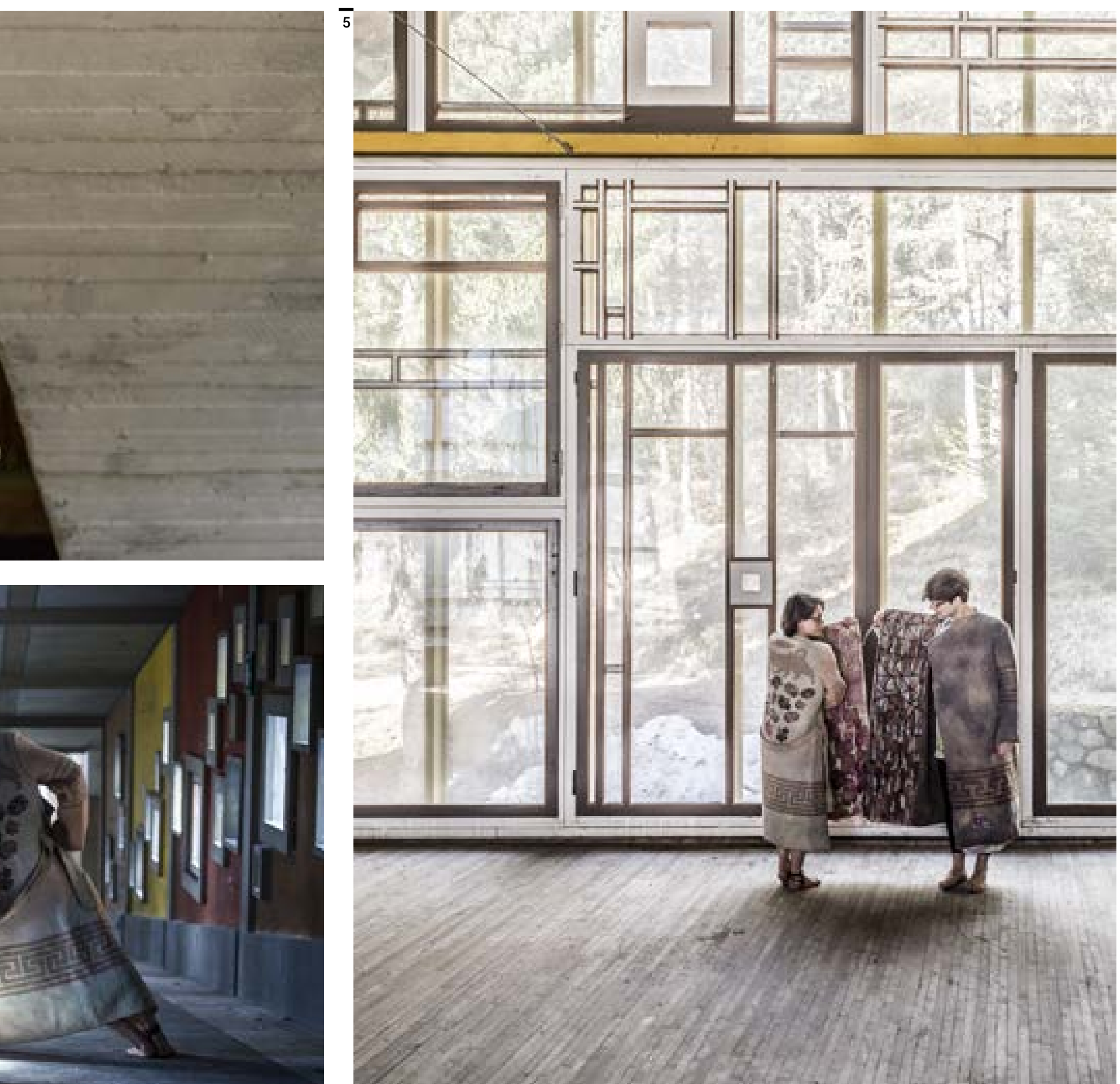
DC opera in (e dal) territorio dolomitico.

Oggi, annoveriamo tra i nostri partner circa cinquecento soggetti, assai eterogenei: politici, amministrativi, produttivi, e poi istituti di ricerca e università, enti di sviluppo, e così via.

Ma, quando si inizia ad operare, prima di tutto viene l'arte contemporanea: vengono gli artisti, armati della loro concretezza trasvalutativa.

Sissignore, primi gli artisti. Questi siti hanno bisogno di cura, e di uno sguardo profondo e franco, che faccia pulizia di definizioni statiche e sentenze (funebri).

L'arte ripensa sempre tutto: non si accontenta di una definizione già data, ed in ciò, spesso, pigra, ferma.
L'arte può essere intesa come una superiore facoltà di stimolo, se è concepita come arma e tecnica di un approccio trasformativo, e non come una patetica occupazione ricreativa esornativa.

Nel modulo funzionale, l'arte non decora, si oppone invece all'assenza di decoro. Perché la trascuratezza è sempre indecorosa, la carenza d'idee, le visioni convenzionali, tra le peggiori povertà dell'uomo, insieme alla banalità e alla bêtise, che ne son figlie.

Diciamo allora che, per poter rivalutare, occorre prima saper travalicare.

Come potremmo pretendere di affrontare un'impresa di trasformazione identitaria, una politica

\section{$\overline{6}$} 6

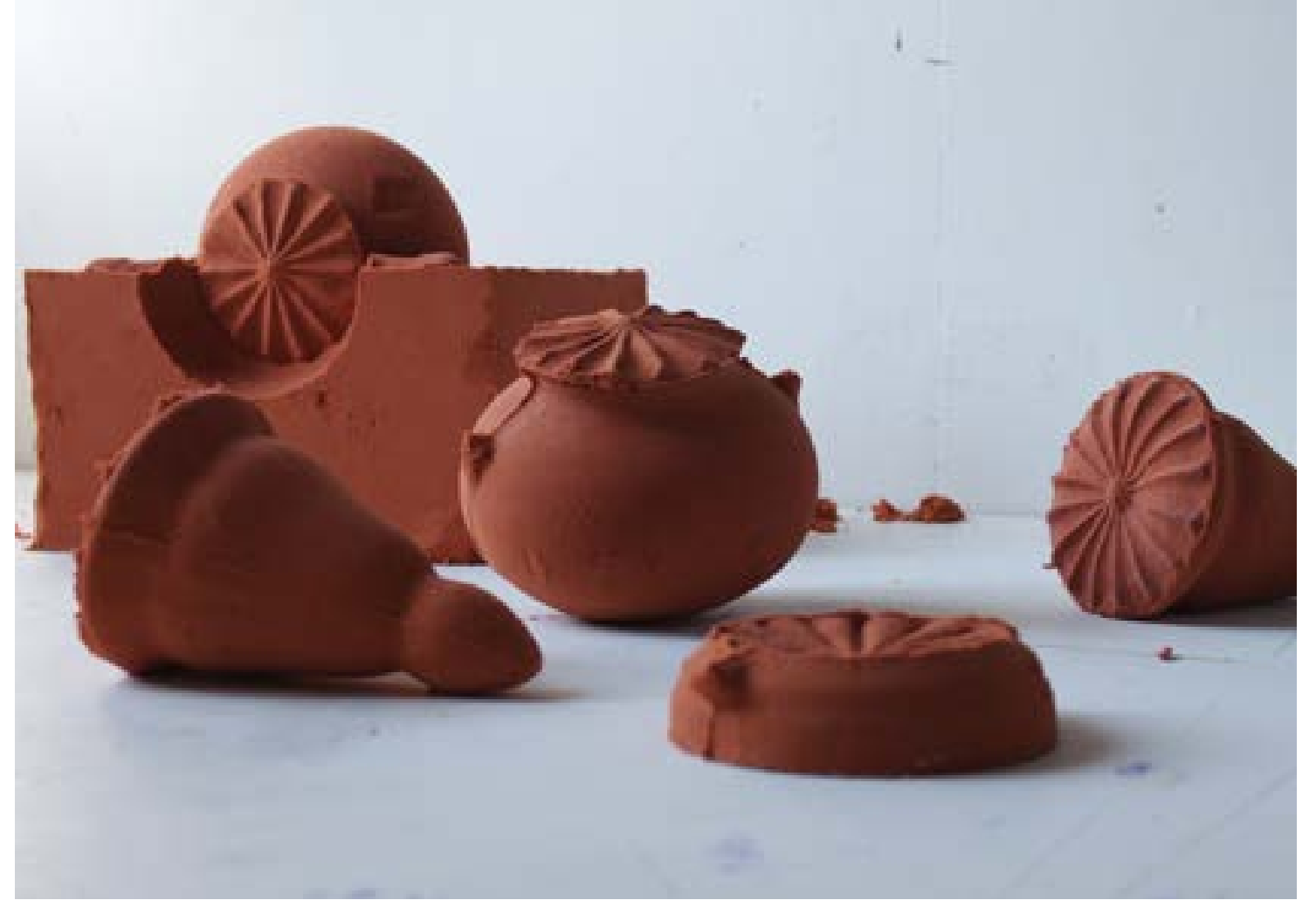

\section{Marta Allegri}

Il lavoro di Marta Allegri accentua, senza esaltarlo (l'arte non è sempre dionisiaca), il rapporto tra il patrimonio valoriale dell'architettura, in particolare quella storica, stinta, corrotta, indisponibile (se non alla memoria: che spesso genera retroversione e paralisi), il pensiero trasformativo, l'azione rigeneratrice della cura, la forma quale manifestazione plastica della rivalutazione.

L'aggrappo al reale (resti, lacerti, lasciti, pietre, muri), si compie attraverso la rielaborazione della materia, la costruzione di una nuova foggia, che non è urna né trina, ma riprocessazione dell'entità, e della sua virtù (sospesa, perduta). È assai solido e netto, questo lavoro leggero (l'arte non è sempre olimpica). È assai concreta, questa visione. È distesa, semplice, chiara. Si tratta di riplasmare i materiali basici (fisicità del concetto). Non di spolverare gli argenti di famiglia (il patrimonio cosiddetto).

L'architettura è la buona pratica costruttiva di un pensiero corretto, aperto, libero, concreto. 
rinnovativa della gestione della risorsa, semplicemente rimanendo seduti su categorie atrofizzate? La montagna, abbiamo detto, non va divorata. Va nutrita, in modo tale che, ad un certo punto, essa possa ricominciare a rilasciare principi nutritivi.

La montagna è il suo farsi, i suoi paesaggi vanno costantemente costruiti, e ricostruiti.

È una costruzione culturale, critica e responsabile. La montagna si muove, non è eterna, non un'invariante fisica: spesso frana.

Nessun valore prescinde dall'uso, quindi nessun valore può essere dato una volta per tutte.

Occorre ridiscutere.

Valori e funzioni cambiano, si esauriscono.
Se questo è vero, servono nuovi inizi, nuovi sguardi, nuove visioni, altri impulsi.

Ma per capire uno spazio, è necessario conoscerlo, e per conoscerlo, bisogna viverlo.

Ecco perché l'istituto della Residenza è imprescindibile: esso consente di lavorare dall'interno.

In tutto ciò, la funzione dell'arte non è solo metaforica, o metasemica.

Non è nemmeno una tecnica dell'invenzione: piuttosto, azione metamorfica.

Non fornisce griglie definitive, ma griglie di riassestamento: la sua mappatura concettuale è dinamica, la pratica l'aggrappa al reale.

Consente di reinterpretare, di visualizzare e di avviare, nuovi cicli e ricicli.

Fig. 6

Marta Allegri, "Forti senza aderenza", polvere di mattone, 2018, Forte Monte Ricco (foto Nicola Noro)

Fig. 7

Un lavoro dell'artista Marta Allegri in Progettoborca L'opera a terra riflette sugli elementi costitutivi dell'architettura. L'installazione si trova negli ex alloggi delle religiose, nella Colonia di Borca, dove Allegri sviluppa un progetto in continuità dal 2015

(foto Giacomo De Donà).

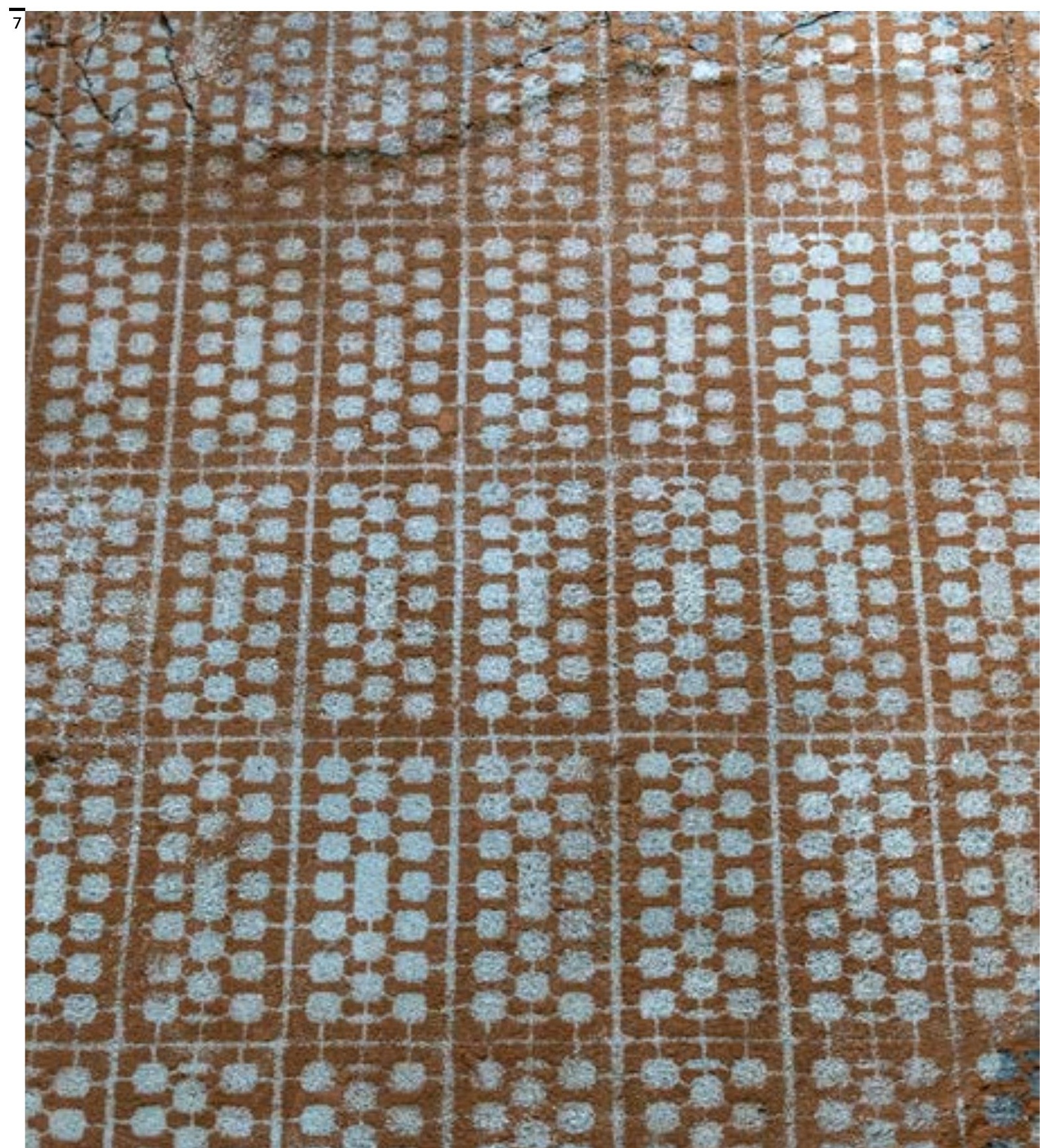


Se i siti sono smorti, se la montagna è ferma: occorre sommuovere.

Non attraverso il cascame culturale. Attraverso la sperimentazione di cantiere, in ambiente.

In questo quadro, l'arte contemporanea, nella realtà e nella dichiarazione, è per noi al centro.

L'arte che smuove la terra e i cieli, che è una prova della capacità di ragione e sentimento, se è concepita come una tecnica reattiva, uno strumento dell'intelligenza e del lavoro e della rivoluzione.

L'arte è una ghigliottina: taglia tutte le teste vuote (se sono le teste di chi male conduce o amministra i territori, si tratta di capi indecorosi).

Si tratta in definitiva di riportare l'arte tra le concrete cose terrene, dove è sempre stata, manifestando le migliori virtù costruttive dell'uomo. Chi la ritiene cosa superflua, è perlomeno ignorante, quindi incapace di prospettive di sorta, e inabile al progetto.

Io stesso sono un curatore d'arte contemporanea e un critico, mica un ricercatore di management dell'arte e della cultura.

Per esser precisi, all'inizio del processo servono questi elementi: il nostro intento rivalutatore, che è chiaro e deciso; il sostegno del Sindaco, e la fiducia della rete territoriale; quindi gli artisti, che sull'eterogenea e aperta piattaforma di condivisione così costituita, cominciano operosamente a dispensare le intelligenze della sensibilità.

Gli artisti, con i curatori spaziali (curare l'identità dello Spazio è l'obiettivo, non allestire le esposizioni nelle scatole sfitte), avviano il processo enzimatico, inaugurando una fase trasformativa, che deve sovvertire l'inerzia, avviando una reazione, e catalizzando le attenzioni.

Marta Allegri, Come si può avviarlo, un simile processo? Con "Braintooling" (foto Giacomo De Donà).

Fig. 9

II nuovo spazio di Casso al Vajont (foto Giacomo De Donà).

Fig. 10

Progettoborca. La Summer

School "Dolomiti

Digital Camp",

un'esperienza

di formazione

realizzata all'ex

Villaggio Eni grazie a una collaborazione

tra Dolomiti

Contemporanee

e H-Farm (foto

Giacomo De Donà). le parole, con un bel convegno d'archeologi e storici, tra sentenze e ricette? O coi progetti di restauro?

No. La pratica non è un Convegno, la coltivazione non è una discussione teorica, né la tavola di un architetto.

Molte altre tecniche e strategie della cultura d'innovazione vengono utilizzate e attuate, insieme all'arte, in questo nostro progetto ampio, antischematico, antisettoriale: esse collaborano alla riattivazione dei siti o delle aree depressi, o generano proattività a tal fine.

Tutto ciò: le idee, l'arte, il cantierismo culturale, le attività laboratoriali e produttive sperimentali, e poi i paesaggisti, gli economisti della cultura, gli architetti sbalzatori, gli intellettuali da campo, questo serve.

Tutto ciò può e deve contribuire a generare una consapevolezza rinnovata, delle identità, dei valori e dei bisogni.

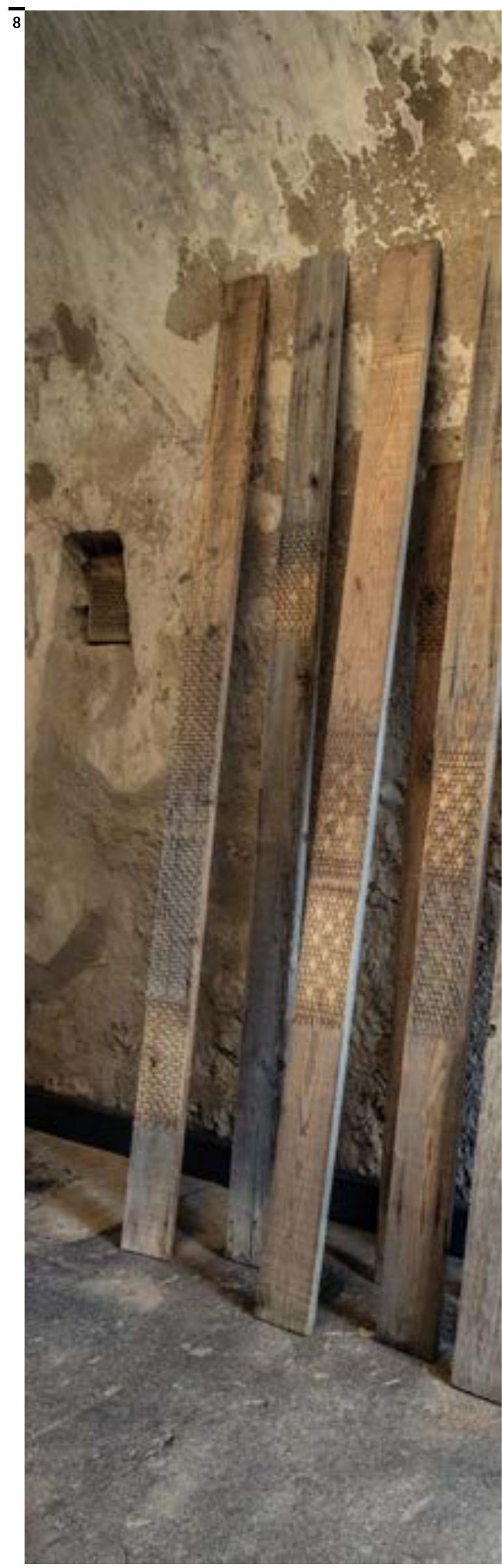


Alcuni casi.

Nel 2012, abbiamo avviato un cantiere sperimentale nell'area del Vajont, trasformando l'ex scuola elementare di Casso, chiusa dal 9 ottobre del 1963, in un Centro per la Cultura della Montagna e del Paesaggio. Con il Concorso Internazionale Twocalls (www.twocalls.net), si trasforma la Diga del Vajont, simbolo della tragedia, in un elemento rinnovativo di un paesaggio mutile.

Nel 2014, abbiamo avviato Progettoborca (www. progettoborca.net), piattaforma di rigenerazione che ha trasformato la gigantesca Colonia dell'ex Villaggio Eni di Borca di Cadore, realizzato dagli anni Cinquanta dall'architetto Edoardo Gellner, come parte del dispositivo di welfare voluto da Enrico Mattei, in un grande, multiverso cantiere della produzione artistica e culturale.

Queste due esperienze, a differenza di altre che abbiamo svolto in modo più rapido (focus), sono strutturali, e di medio-lungo periodo.

Questo perché, evidentemente, i siti del Vajont e di Borca possiedono una tale rilevanza e un tale peso specifico, che sarebbe impensabile affrontarne l'identità, volendo incidere su di essa, in un lasso di tempo breve. Occorre un'azione forte, e occorre che essa sia insistita.

Nei siti, in ogni sito, viene attivata una Residenza internazionale, in grado di accogliere centinaia di artisti da tutto il mondo, che lavorano (insieme a paesaggisti, architetti, designer, scienziati forestali, atenei, istituti di ricerca, territorio), secondo programmi e concept che sempre intavolano sulle peculiarità territoriali e d'area, sull'identità dei siti stessi e della montagna, su temi capitali.

Come ad esempio Tempesta Vaia, l'evento distruttivo che ad ottobre 2018 ha devastato i boschi delle Dolomiti e del triveneto, e che impone di interrogarsi su temi grandi, come la rigenerazione ambientale e i cambiamenti climatici.

Oppure, per rimanere a Borca, come nel caso della Candidatura di Milano e Cortina alle Olimpiadi inver- nali del 2026, all'interno del cui dossier DC propone l'idea del recupero dell'ex Villaggio Eni come parte dell'infrastruttura logistica necessaria alla manifestazione, in una chiave di rigenerazione territoriale, che guardi ben oltre alle necessità del mero evento sportivo.

Diciamo che, rispetto alla disponibilità di questi siti-risorsa, inabissati o semisommersi, non si ragiona mai in termini di perdita o di lutto, ma sempre di opportunità, potenzialità e necessità.

Questi siti eccezionali, sono nati, hanno trainato, si sono fermati.

Se vogliamo iniziare, per essi e per noi, qualcosa di nuovo, che abbia senso e valore, se vogliamo riscattarli, dobbiamo svegliarci. Il contemporaneo, se interconnesso ad una strategia delle reti, è un metodo per sciogliere le concrezioni culturali che, come diga, impediscono il rilascio dei valori, la loro riprocessazione, la loro riabilitazione.

DG è leggibile anche come una sorta di dispositivo di rivalutazione azionista, che talvolta persino compie i propri focus (non le proprie strategie) secondo una logica performativa provocatrice, che si oppone, come una pietra in mezzo al sentiero, all'insulsa incapacità, da parte dell'uomo, di bene utilizzare le proprie risorse, di bene abitare la propria terra in valore, di bene concepire le politiche di sviluppo per i territori.

La montagna smuore, si spopola, non è più? Nessun tragico fato, o ineluttabile corso della storia: la colpa è dell'uomo, che agisce male.

Si può (si deve) reagire: alcuni progetti, o pratiche, mostrano vie, che son bandoli e non precetti, con la coltivazione di scene sperimentali alle volte, e di cantieri alti e appuntiti sempre, dove si sciolgono, si svolgono, i flussi trasvalutativi. Dove sorgono le spinte della volontà nella ragione, della responsabilità nella cura, dell'impegno intellettuale. Dove si sa utilizzare la motosega, anche. 
"Giorno di festa",

Installazione su

muro di una vecchia

casa del borgo

Casso, 2015 (foto

Giacomo De Donà)

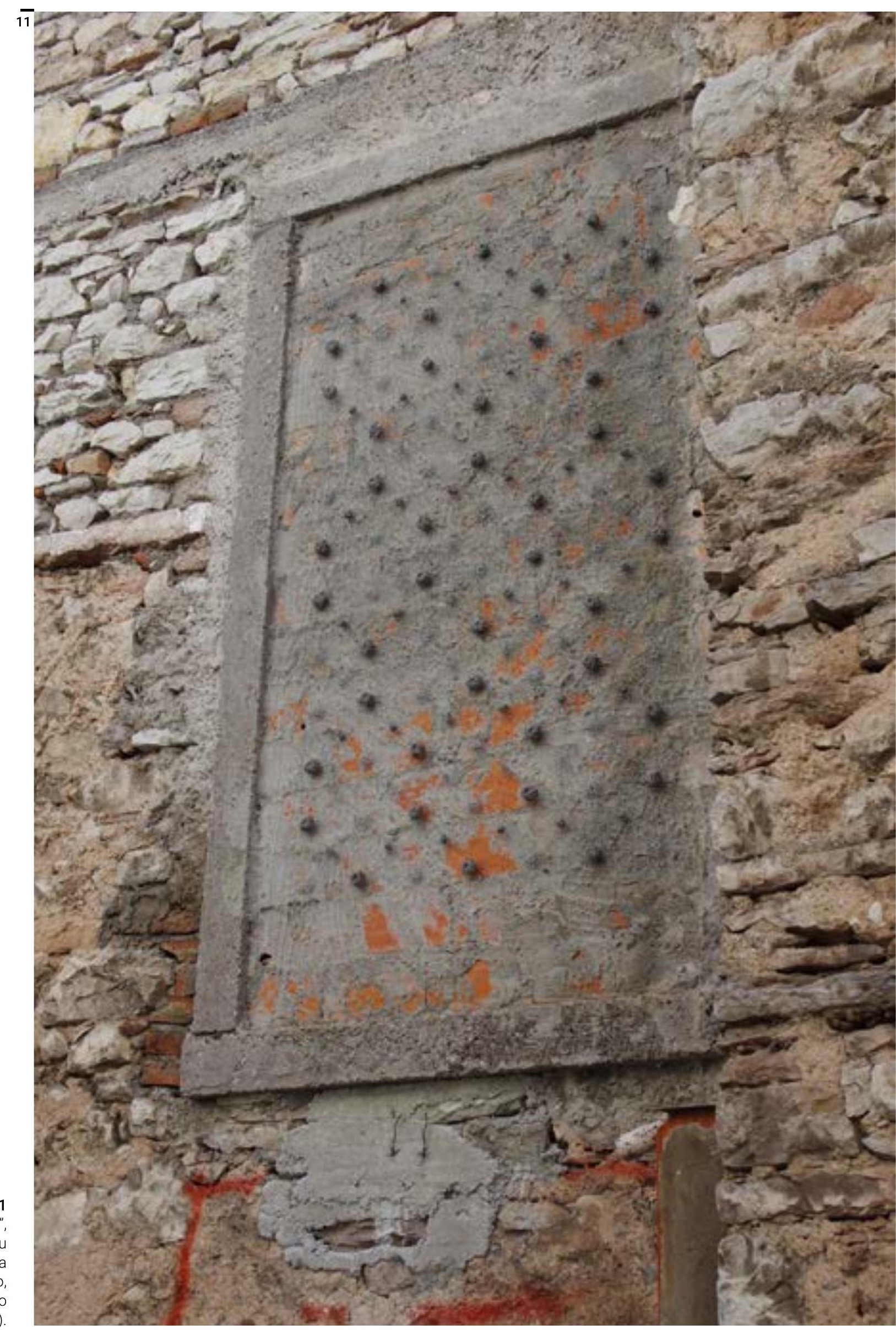

\section{$\overline{11}$}

